\title{
Construction of a Conceptual Framework for Assessment of Health-Related Quality of Life in Dogs With Osteoarthritis
}

\section{OPEN ACCESS}

Edited by:

Jeremy N. Marchant,

Livestock Behavior Research Unit (USDA-ARS), United States

Reviewed by:

Daniel Simon Mills,

University of Lincoln, United Kingdom

David Bruyette,

Anivive Lifesciences, United States

*Correspondence:

Claire Roberts

claire.e.roberts@surrey.ac.uk

Specialty section:

This article was submitted to Animal Behavior and Welfare,

a section of the journal

Frontiers in Veterinary Science

Received: 15 July 2021

Accepted: 31 August 2021

Published: 24 September 2021

Citation:

Roberts C, Armson B, Bartram D, Belshaw Z, Capon H, Cherry G, Gonzalez Villeta L, McIntyre SL,

Odeyemi I and Cook AJC (2021)

Construction of a Conceptual

Framework for Assessment of Health-Related Quality of Life in Dogs With Osteoarthritis.

Front. Vet. Sci. 8:741864. doi: 10.3389/fvets.2021.741864

\begin{abstract}
Claire Roberts ${ }^{1 *}$, Bryony Armson ${ }^{1}$, David Bartram ${ }^{2}$, Zoe Belshaw ${ }^{3}$, Hannah Capon ${ }^{4}$, Georgina Cherry ${ }^{1}$, Laura Gonzalez Villeta ${ }^{1}$, Shona L. McIntyre ${ }^{1}$, Isaac Odeyemi ${ }^{2}$ and Alasdair J. C. Cook ${ }^{1}$

${ }^{1}$ vHive, School of Veterinary Medicine, University of Surrey, Guildford, United Kingdom, ${ }^{2}$ Outcomes Research, Zoetis, Loughlinstown, County Dublin, Ireland, ${ }^{3}$ EviVet Research Consultancy, Nottingham, United Kingdom, ${ }^{4}$ Canine Arthritis Management, Shoreham-by-Sea, United Kingdom
\end{abstract}

An owner's ability to detect changes in the behavior of a dog afflicted with osteoarthritis $(\mathrm{OA})$ may be a barrier to presentation, clinical diagnosis and initiation of treatment. Management of $O A$ also relies upon an owner's ability to accurately monitor improvement following a trial period of pain relief. The changes in behavior that are associated with the onset and relief of pain from OA can be assessed to determine the dog's health-related quality of life (HRQOL). HRQOL assessments are widely used in human medicine and if developed correctly can be used in the monitoring of disease and in clinical trials. This study followed established guidelines to construct a conceptual framework of indicators of $H R Q O L$ in dogs with OA. This generated items that can be used to develop a $\mathrm{HRQOL}$ assessment tool specific to dogs with OA. A systematic review was conducted using Web of Science, PubMed and Scopus with search terms related to indicators of $\mathrm{HRQOL}$ in dogs with osteoarthritis. Eligibility and quality assessment criteria were applied. Data were extracted from eligible studies using a comprehensive data charting table. Resulting domains and items were assessed at a half-day workshop attended by experts in canine osteoarthritis and quality of life. Domains and their interactions were finalized and a visual representation of the conceptual framework was produced. A total of 1,264 unique articles were generated in the database searches and assessed for inclusion. Of these, 21 progressed to data extraction. After combining synonyms, 47 unique items were categorized across six domains. Review of the six domains by the expert panel resulted in their reduction to four: physical appearance, capability, behavior, and mood. All four categories were deemed to be influenced by pain from osteoarthritis. Capability, mood, and behavior were all hypothesized to impact on each other while physical appearance was impacted by, but did not impact upon, the other domains. The framework has potential application to inform the development of valid and reliable instruments to operationalize measurement of $\mathrm{HRQOL}$ in canine OA for use in general veterinary practice to guide $\mathrm{OA}$ management decisions and in clinical studies to evaluate treatment outcomes.

Keywords: osteoarthritis, conceptual framework, quality of life, degenerative joint disease, dog, pain, canine 


\section{INTRODUCTION}

Osteoarthritis (OA), also known as degenerative joint disease, refers to the irreversible degeneration of cartilage and other tissues within joints. Prevalence estimates in the late 1990s have been as high as $20-30 \%$ of dogs over the age of 1 year $(1,2)$. A recent study using a canine OA screening checklist indicated that $37 \%$ of screened dogs had clinical signs associated with OA (3). OA can occur in any dog although it is more often diagnosed in older dogs and dogs of certain large breeds (4). Pain associated with OA has a broad impact in dogs through its effects on gait and movement, function, affective state, social relationships and sleep (5). Foundational to OA treatment is the proactive management of pain over the course of the disease to help ensure the success of other supportive care (1). UK veterinarians rate OA to be the most common cause of pain in dogs (6).

Owners may not recognize signs of OA that are presented by their dogs and this can be a major barrier to initiation of treatment (7). Case management often involves a trial period of pain relief treatment (7), the evaluation of which relies on owners monitoring changes accurately and noticing any improvements to quality of life (QOL). Quality of life related to a disease, or health-related quality of life (HRQOL), in dogs can be defined as "in the context of an altered health state and associated health interventions, the evaluation by the individual of its circumstances (internal and external), and the affective (emotional) response to those circumstances" (8). Humans with osteoarthritis have reported that it causes significantly lower levels of quality of life (9-13). It is likely that $\mathrm{OA}$ affects numerous aspects of quality of life in dogs $(5,14-16)$.

Humans are generally able to assess their own quality of life. This may be performed using a patient-reported outcome measure (PROM), an instrument that allows the patient to report on aspects of their own health, such as pain and QOL $(17,18)$. Non-human animals are unable to report their own health outcome and require a proxy, which for dogs is generally the owner. Generic and disease specific HRQOL measures have been developed for dogs (19-22). The use of both approaches has been reported in veterinary literature (23). Disease-specific measures of QOL have the advantage of frequently being more responsive (sensitivity to detect change over time) and clinically useful than generic QOL measures which do not focus on any specific condition (24) while generic measures have the advantage of enabling comparisons of QOL burden and treatment benefit across diseases (25).

HRQOL assessments are widely used in human medicine. The Food and Drug Administration (FDA) patient-reported outcome (PRO) guidance outlines methods for the development and validation of human PROs and HRQOL measures that can be used to support claims in medical product labelling (26). This guidance recommends the initial development of a conceptual framework (CF), which is a model that identifies the QOL domains that are affected by the health-related condition of interest and the relationship between them. This paper presents a $\mathrm{CF}$ for HRQOL in dogs with $\mathrm{OA}$. The $\mathrm{CF}$ will be used to generate initial items for a PROM instrument. Conceptual frameworks have already been used to inform the development and comprehensiveness of companion animal QOL measures (27).

The current study aimed to construct a conceptual framework of indicators of HRQOL in dogs with OA, focusing on the subjective experience of the dog. The CF was based upon a systematic literature review that was reviewed by an expert panel. The framework has potential application to inform the development of valid and reliable instruments to operationalize measurement of HRQOL in canine OA for use in general veterinary practice to guide $\mathrm{OA}$ management decisions and in clinical studies to evaluate treatment outcomes.

\section{MATERIALS AND METHODS}

\section{Literature Search}

A systematic literature review was performed in August 2020 following the Preferred Reporting Items for Systematic Reviews and Meta-analyses (PRISMA) guidelines (28). Three scientific databases (Web of Science, PubMed and Scopus) were searched with the terms [("dog" OR "canine") AND ("degenerative joint disease" OR "osteoarthritis" OR "musculoskeletal") AND ("quality of life" OR "pain") AND ("indicator" OR "measure" OR "sign" OR "symptom")]. Duplicate articles were removed. Remaining studies were assessed for inclusion by multiple authors (BA, GC, SLM, LGV) using the following predefined eligibility criteria: (i) focused on dogs (ii) published in or after the year 2000, (iii) available in the English language, (iv) peer reviewed (v) original research and (vi) focused on indicators of chronic musculoskeletal pain or its impact on quality of life. Studies concerning acute pain, risk factors for pain or on treatment effectiveness were excluded (Figure 1). Studies were initially assessed by screening of the title and the abstract; remaining articles were assessed by reading the full article. The bibliographies of eligible search result articles were checked for further additional eligible articles.

Quality assessment was performed using a modified version of the STROBE (Strengthening The Reporting of Observational studies in Epidemiology) guidelines (29). This consisted of twelve criteria (Supplementary Table 1). Articles meeting six or more of the criteria were eligible for progression to data extraction. Bias of risk within studies was addressed within the quality assessment.

\section{Data Extraction}

Indicators of osteoarthritis and/or its impact on quality of life were extracted to generate "items" from each study. Only indicators listed within the body of the manuscript were included. Data were initially extracted by one of four authors (BA, GC, SLM, LGV), with the process repeated independently by a single author (CR) for all studies. A comprehensive data charting table based on a previous review for HRQOL in cats with osteoarthritis (30) and refined iteratively was used to extract relevant information from included sources of evidence. This consisted of nine domains: mobility, physical appearance, energy/vitality/behavior, temperament, pain expressions, sociability and well-being. Items were cross checked between studies to identify duplications, and the frequencies of each item were recorded. Items that 
Records identified using the search term:

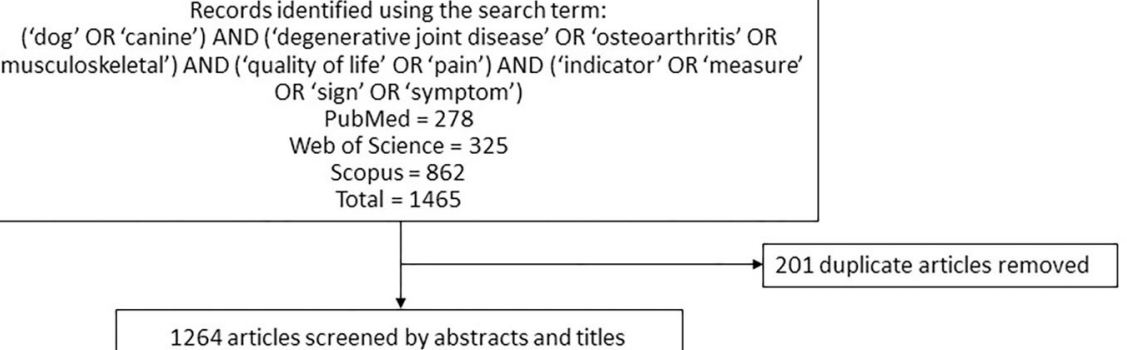

1264 articles screened by abstracts and titles



FIGURE 1 | Flow diagram summarizing the literature search on canine osteoarthritis and application of eligibility criteria.

were deemed to be synonyms (e.g., "weary" and "tired") were combined by the authors as applicable.

\section{Expert Workshop}

The resulting domains and items were assessed at a workshop attended by members of the research team, a specialist in small animal internal medicine with a $\mathrm{PhD}$ in decision making in dogs with osteoarthritis and a veterinary surgeon who founded an initiative to provide advice and education on canine arthritis to owners and veterinary professionals. Prior to the meeting, panel attendees were provided with a PowerPoint file covering the methodology and results including a table of key domains/items (Supplementary Table 2) and a brief for the meeting. The meeting brief was to assess the appropriateness of nomenclature for domains, whether each of the items were in the "correct" domain and whether any of the items could be combined (i.e., synonymous). The brief also stated the aim of the meeting which was to establish a hypothesis for how the domains interrelate and to prepare a diagram to illustrate the domains and their interactions.

The workshop took place in November 2020 online and lasted 2 1/2 h. Domains were discussed and final titles decided, with each item assessed for designation to the correct domain. Additionally, any potential missing items were discussed. Hypothesized directional interactions between the domains were identified and a visual representation of the conceptual framework was produced.

\section{RESULTS}

A total of 1,264 unique articles were generated in the database searches and assessed for inclusion using the title and abstract (Figure 1). Of these, 76 progressed to have the full manuscript screened. Two articles were added from reference lists $(14,31)$. Twenty-one studies were identified to be eligible for inclusion in the data extraction process $(8,14-16,31-48)$. Quality assessment allowed all 21 to progress (Supplementary Table 1).

The eligible studies dated from 2003 to August 2020 inclusive. Study characteristics are shown in Supplementary Table 3. All studies bar one (45) addressed the risk of bias. Sixteen (76.2\%) of the studies described the development $(n=11)$, translation $(n$ $=2)$, or use $(n=3)$ of pain or HRQOL assessment instrument[s]. There were five instruments: the Liverpool Osteoarthritis in Dogs (LOAD) score, $(31,46)$, Canine Brief Pain Inventory (CBPI), (15, 38, 39, 44, 46), Helsinki Chronic Pain Index (HCPI) (16, 38, 41, 42, 46), the Canine Orthopedic Index (COI) (32, 33, $36,37)$ and the Glasgow University health-related dog behavior questionnaire (GUVQuest) $(8,45,48)$.

Data extraction produced 134 unique items (Supplementary Table 2) categorized across domains as follows: physical appearance six items (4.5\%); mobility 28 items (20.9\%); energy/vitality/behavior 35 items (26.1\%); temperament 47 items (35.1\%); pain expression 11 items (8.2\%); sociability 7 items (5.2\%). The domain "well-being" was removed from the list of domains as all items within well-being could also be categorized within one of the other domains. The most common items (in six or more studies) were: rising from lying, climbing 
TABLE 1 | Domains and items resulting from a literature review on indicators of HRQOL in dogs with osteoarthritis, after categorization by an expert panel.

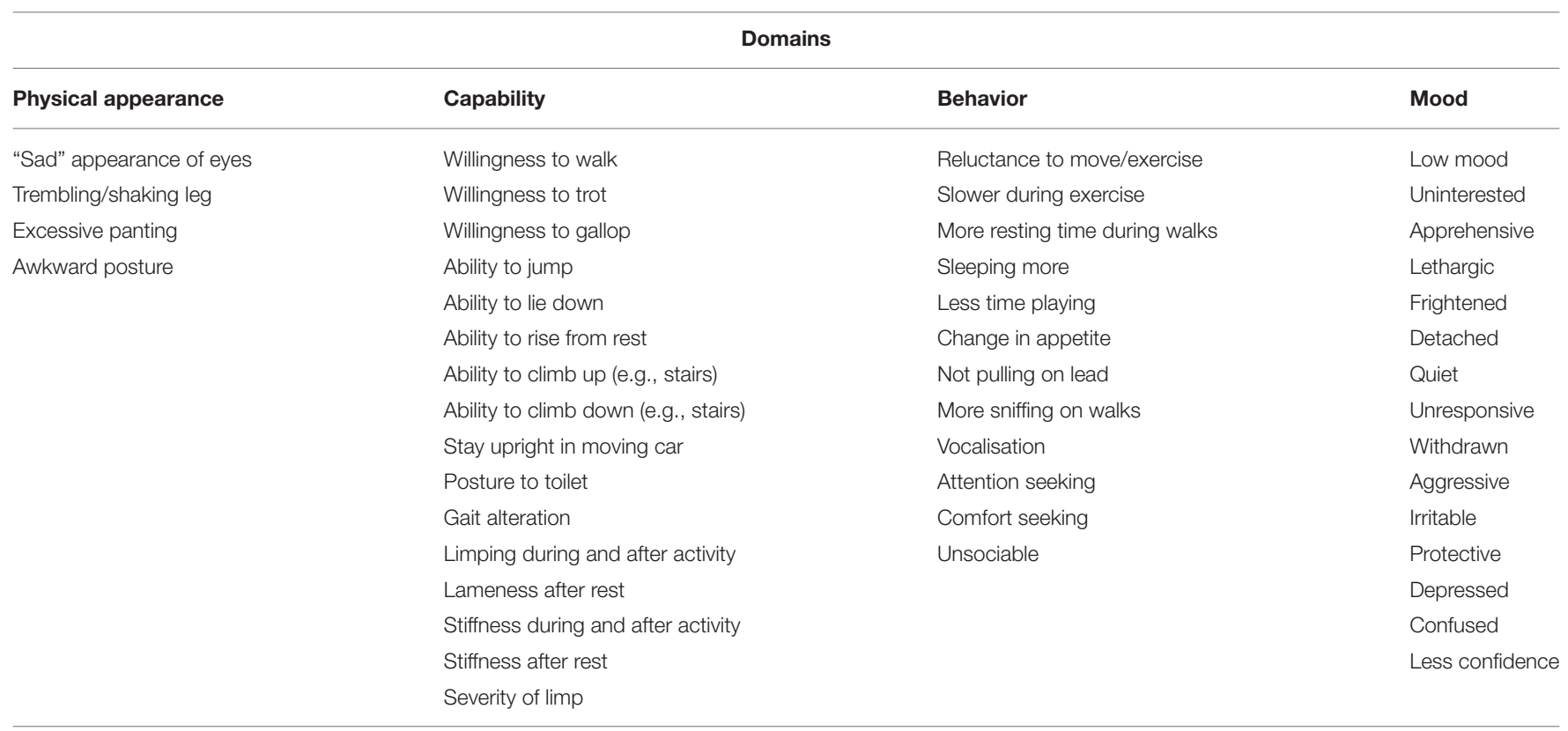

up, vocalization, climbing down, jumping up, jumping down and general activity. Over half of the items were unique to a single study $(77 / 134 ; 57.5 \%)$. Items ranged from $0-59$ per study, with an average of 12.5 items per study. Combining synonyms reduced the number of items to 47 .

The expert workshop resulted in four remaining categories: physical appearance, capability, behavior, and mood (Table 1). The original domain "physical appearance" was retained as a heading. "Mobility" was renamed "capability" to reflect the ability of a dog to perform certain tasks not necessarily related to moving, such as staying upright in a car. The domain "energy, vitality and behavior" was shortened to "behavior" and was thought to incorporate all the items previously within the "sociability" domain, such as attention seeking. "Temperament" was renamed "mood" after the top item within the domain and to reflect that the items in the domain can be either positive or negative. The items within the domain "pain expressions" were incorporated into other domains, as all items were deemed to be reactions to pain.

The four domains were incorporated into a visual conceptual framework model (Figure 2). All four categories were deemed to be influenced by pain from osteoarthritis. Hypothesized interactions between the domains are displayed in Table 2.

\section{DISCUSSION}

The current study aimed to construct a conceptual framework of indicators of HRQOL in dogs with OA, through a systematic literature review and expert panel, focusing on the subjective experience of the dog. This approach is consistent with FDA guidance (26) for the development of PROMs. The literature search revealed 21 quality, peer-reviewed studies relating to canine OA and its impact on QOL.
Over half of the studies described the development of one of five existing instruments or their translation into other languages. These instruments are varied in their relation to HRQOL in dogs with osteoarthritis. The $\operatorname{LOAD}(31)$ and $\operatorname{HCPI}(16,42)$ instruments both focus on orthopedic pain and its impact on exercise and mobility rather than HRQOL, although the HCPI does incorporate one question each on mood and vocalization. The CBPI $(15)$ and COI $(36,37)$ were also developed with the aim of assessing the impact of pain and also focus on mobility. The CBPI includes a question relating to enjoyment of life and both incorporate a global impression of QOL. However, neither include items relating to physical appearance or sociability of the dog and the COI does not refer to mood. The current study focused on measuring the experiences of dogs with osteoarthritis relating to their day-to-day life, rather than clinical signs of OA and severity of pain, allowing a more global HRQOL measurement than these earlier instruments.

More relevant to HRQOL was the Glasgow University healthrelated dog behavior questionnaire (GUVQuest) which was specifically developed to assess the impact of pain, including from OA, on HRQOL $(8,45,48)$. This instrument appears comprehensive and includes 109 descriptor items that reflect subjective experience, each with an associated 7-point Likert-type rating scale on which responses are framed on an agree-disagree continuum. Factor analysis revealed a range of 12 purported HRQOL domains $(8,45,48)$. However, the instrument is timeconsuming to complete and has since been shortened to a 46 -item measure of generic (rather than OA-specific) HRQOL (45).

Three studies used one of these instruments, with one performing a welfare assessment of a population of dogs (33). A further study used the HCPI to assess the use of mechanical thresholds in joints of dogs (41), and the other tested whether medication biased the response of owners when assessing the pain of their dogs with OA (38). This demonstrates 
Examples:

- Excessive panting

- Trembling leg

- Abnormal posture

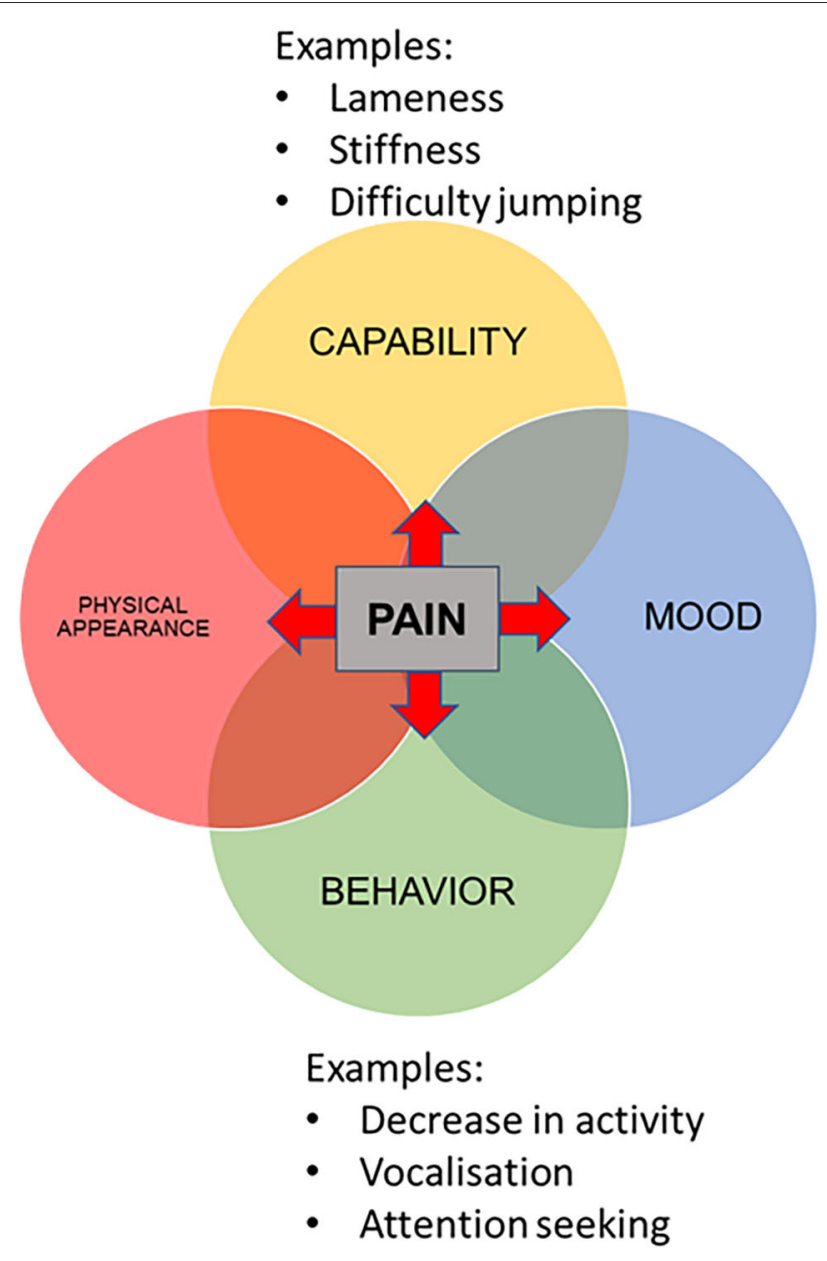

Examples:

- Difficultyjumping

\section{CAPABILITY}

BEHAVIOR

\section{Examples:}

- Decrease in activity

- Attention seeking

FIGURE 2 | Conceptual framework for the assessment of health-related quality of life in dogs with osteoarthritis, including three example items in each domain.

TABLE 2 | Hypothesised direction of interactions between the four domains represented in the conceptual framework for HRQOL in dogs with osteoarthritis (Figure 2).



the range of these clinical instruments but indicates a low level of use, although it is possible that further use of these instruments has not been reported. Similarly, there are few published studies that have used other canine QOL assessment tools (19).

Of the remaining six studies, two reported clinical-based methods to OA assessment: mechanical joint threshold (43) and thermal sensory testing (47). Although perhaps relevant to the diagnosis and monitoring of OA, these are not ownerreported measures and therefore provided no useful items to the CF in the current study. One study used clinical records to retrospectively investigate aggressive behaviors in dogs with osteoarthritis (34). This focus on behavior provided useful additions to the conceptual framework that the studies using the existing instruments, with their focus on mobility and exercise, may not have revealed. The final two studies used qualitative methods, interviewing owners of osteoarthritic dogs on their experiences $(14,35)$. These also provided useful items that may have been missed by the quantitative studies, such as dogs having slower and shorter walks (14). 
More than half of the items were unique to a single manuscript. This was especially the case in the original domains of "energy" and "temperament" and perhaps reflects the subjectivity of these domains. There was scope for different semantic interpretations for several items because their exact meaning within the context of their respective source articles was not always clearly characterized. Combination of synonyms reduced the number of items from 134 to 47 . The mobility domain had fewer unique items and contained most of the most commonly reported items. There are several potential reasons for this. It could indeed be that these are the most common issues in OA in dogs, either as the most important to owners, or the most obvious signs. This is reflected in the clinical signs reported to be useful in a presumptive OA diagnosis (49). However, aspects of mobility were strongly represented in the existing instruments, which were developed or used in nearly three quarters of the studies screened in this review.

The final conceptual framework comprised four domains representing HRQOL of dogs with osteoarthritis. The final domains were mobility, behavior, mood, and physical appearance which were all deemed to be affected by pain. A negative impact of pain on QOL has been reported in humans (50-52). It is likely that this is similar in dogs; chronic pain has a wide range of impacts including loss or difficulty in expression of normal behaviors, such as using stairs and the development of new behaviors $(53,54)$. Other domains were hypothesized to all impact on each other, apart from physical appearance which was impacted on by capability, behavior, and mood but did not itself have any impact on the other domains. This is an indication of the complex nature of quality of life and its assessment.

The resulting CF is consistent with a non-peer reviewed model produced by Canine Arthritis Management (CAM) representing the impact of chronic pain on capability, behavior, muscular changes and posture (55). Although a founder of CAM was present at the workshop in this study, the similarity of the models was noticed after the majority of construction of the CF was completed. Perhaps the main difference is the presence of the "mood" domain in the CF. It is likely that mood is included under the domain "behavior" in the CAM model. Results are also consistent with recent reports on a conceptual framework of HRQOL in cats with osteoarthritis using a similar methodology to the current study (30). The main difference was the removal of a "well-being" category in the current study. There were no items found to be included under "well-being" that could not be categorized under one of the other domains. There is not currently a clear distinction between the terms "well-being" and "quality of life" and it is likely that the terms overlap or are even synonymous (56). If this were the case, this would support this domain being incorporated into the other categories.

There were some limitations to this study, perhaps the main one being that the multiple references to existing pain and HRQOL instruments in the screened papers, which limited the usefulness of quantification of item frequency in this review. This may have resulted in an overestimation of the importance of mobility and exercise-based items. However, the discussion from the expert panel allowed the review of these items and mobility and exercise (termed "capability") were deemed to be important.
There may also have been bias at the review level by searching publications only in the English language. Again, the use of an expert panel allowed for the addition of any items that may have been missed in the review and no items were added.

The conceptual framework developed by this study has highlighted the complexity of HRQOL in dogs with osteoarthritis, and the impact of pain on all other HRQOL domains. It can be used as a first step in the development of a disease-specific instrument to measure HRQOL in dogs with osteoarthritis, in order to encourage and better monitor treatment. A future qualitative concept elicitation study with key informants including veterinarians and dog owners would provide additional evidence to validate whether the HRQOL domains and their interrelations in our model reflect real-world experiences.

\section{Resource Identification Initiative}

Europe PubMed Central, RRID:SCR_005901

\section{DATA AVAILABILITY STATEMENT}

The original contributions presented in the study are included in the article/Supplementary Material, further inquiries can be directed to the corresponding authors.

\section{AUTHOR CONTRIBUTIONS}

$\mathrm{AC}, \mathrm{DB}$, and $\mathrm{IO}$ contributed to conception and design of the study. BA performed the literature search. BA, GC, SM, and LG performed sifting, data extraction, and quality assessment. Data extraction was audited by CR. AC, BA, CR, and DB planned and attended the expert workshop. $\mathrm{ZB}$ and $\mathrm{HC}$ provided expert advice at the workshop. CR wrote the first draft of the manuscript. BA wrote sections of the manuscript. All authors contributed to manuscript revision, read, and approved the submitted version.

\section{FUNDING}

The authors declare that this study received funding from Zoetis under the auspices of a wider collaboration: the Veterinary Health Innovation Engine (vHive). In addition to the funding described above (Conflicts of Interest), the funder had the following involvement in the study: compensation to expert panel.

\section{ACKNOWLEDGMENTS}

The authors would like to acknowledge Adam Trish for project management at vHive, the University of Surrey.

\section{SUPPLEMENTARY MATERIAL}

The Supplementary Material for this article can be found online at: https://www.frontiersin.org/articles/10.3389/fvets. 2021.741864/full\#supplementary-material 


\section{REFERENCES}

1. Johnston SA. Osteoarthritis: joint anatomy, physiology, and pathobiology. Vet Clin North Am Small Anim Pract. (1997) 27:699-723. doi: 10.1016/s0195-5616(97)50076-3

2. Johnson JA, Austin C, Breur GJ. Incidence of canine appendicular musculoskeletal disorders in 16 veterinary teaching hospitals from 1980 through 1989. Vet Comp Orthop Trauma. (1994) 7:56-69. doi: 10.1055/s-0038-1633097

3. Wright A, Amodie D, Cernicchiaro N, Lascelles B, Pavlock A. Diagnosis and treatments rates of osteoarthritis in dogs using a health risk assessment (HRA) or health questionniare for osteoarthritis in general veterinary practice. Value Health. (2019) 22:s387. doi: 10.1016/j.jval.2019.04.1886

4. Anderson KL, O'neill DG, Brodbelt DC, Church DB, Meeson RL, Sargan D, et al. Prevalence, duration and risk factors for appendicular osteoarthritis in a Uk dog population under primary veterinary care. Sci Rep. (2018) 8:5641. doi: 10.1038/s41598-018-23940-z

5. Lascelles BDX, Brown DC, Conzemius MG, Gill M, Oshinsky ML, Sharkey M. Measurement of chronic pain in companion animals: discussions from the pain in animals workshop (PAW) 2017. Vet J. (2019) 250:71-8. doi: 10.1016/j.tvjl.2019.07.001

6. Bell A, Helm J, Reid J. Veterinarians' attitudes to chronic pain in dogs. Vet Rec. (2014) 175:428. doi: 10.1136/vr.102352

7. Belshaw Z, Dean R, Asher L. Could it be osteoarthritis? how dog owners and veterinary surgeons describe identifying canine osteoarthritis in a general practice setting. Prev Vet Med. (2020) 185:105198. doi: 10.1016/j.prevetmed.2020.105198

8. Wiseman-Orr Ml, Scott EM, Reid J, Nolan AM. Validation of a structured questionnaire as an instrument to measure chronic pain in dogs on the basis of effects on health-related quality of life. Am J Vet Res. (2006) 67:1826-36. doi: 10.2460/ajvr.67.11.1826

9. Alkan BM, Fidan F, Tosun A, Ardiçoglu Ö. Quality of life and self-reported disability in patients with knee osteoarthritis. Mod Rheumatol. (2014) 24:16671. doi: 10.3109/14397595.2013.854046

10. Mahir L, Belhaj K, Zahi S, Azanmasso H, Lmidmani F, El Fatimi A. Impact of knee osteoarthritis on the quality of life. Ann Phys Rehabil Med. (2016) 59:e159. doi: 10.1016/j.rehab.2016.07.355

11. Woo J, Lau E, Lee P, Kwok T, Lau WC, Chan C, et al. Impact of osteoarthritis on quality of life in a Hong Kong Chinese population. J Rheumatol. (2004) 31:2433-8. Available online at: https://www.jrheum.org/content/31/ $12 / 2433$

12. Rabenda V, Manette C, Lemmens R, Mariani A-M, Struvay N, Reginster JY. Prevalence and impact of osteoarthritis and osteoporosis on health-related quality of life among active subjects. Aging Clin Exp Res. (2007) 19:55-60. doi: 10.1007/bf03325211

13. Cook C, Pietrobon R, Hegedus E. Osteoarthritis and the impact on quality of life health indicators. Rheumatol Int. (2007) 27:315-21. doi: 10.1007/s00296-006-0269-2

14. Belshaw Z, Dean R, Asher L. Slower, shorter, sadder: a qualitative study exploring how dog walks change when the canine participant develops osteoarthritis. BMC Vet Res. (2020) 16:85. doi: 10.1186/s12917-020-02293-8

15. Brown DC, Boston RC, Coyne JC, Farrar JT. Development and psychometric testing of an instrument designed to measure chronic pain in dogs with osteoarthritis. Am J Vet Res. (2007) 68:631-7. doi: 10.2460/ajvr.68.6.631

16. Hielm-Björkman AK, Rita H, Tulamo R-M. Psychometric testing of the helsinki chronic pain index by completion of a questionnaire in finnish by owners of dogs with chronic signs of pain caused by osteoarthritis. Am J Vet Res. (2009) 70:727-34. doi: 10.2460/ajvr.70.6.727

17. Weldring T, Smith SM. Patient-reported outcomes (PROS) and patientreported outcome measures (PROMS). Health Serv Insights. (2013) 6:61-8. doi: $10.4137 /$ hsi.s11093

18. Mercieca-Bebber R, King MT, Calvert MJ, Stockler MR, Friedlander M. The importance of patient-reported outcomes in clinical trials and strategies for future optimization. Patient Relat Outcome Meas. (2018) 9:353-67. doi: $10.2147 /$ prom.s156279

19. Belshaw Z, Asher L, Harvey N, Dean R. Quality of life assessment in domestic dogs: an evidence-based rapid review. Vet J. (2015) 206:203-12. doi: 10.1016/j.tvjl.2015.07.016
20. Vøls KK, Heden MA, Kristensen AT, Sandøe P. Quality of life assessment in dogs and cats receiving chemotherapy - a review of current methods. Vet Comp Oncol. (2017) 15:684-91. doi: 10.1111/vco.12242

21. Giuffrida MA, Brown DC, Ellenberg SS, Farrar JT. Development and psychometric testing of the canine owner-reported quality of life questionnaire, an instrument designed to measure quality of life in dogs with cancer. J Am Vet Med Assoc. (2018) 252:1073-83. doi: 10.2460/javma.252.9.1073

22. Davies V, Reid J, Wiseman-Orr ML, Scott EM. Optimising outputs from a validated online instrument to measure health-related quality of life (HRQL) in dogs. PLoS ONE. (2019) 14:e0221869. doi: 10.1371/journal.pone.0221869

23. Belshaw Z, Asher L, Dean RS. Systematic review of outcome measures reported in clinical canine osteoarthritis research. Vet Surg. (2016) 45:480-7. doi: 10.1111/vsu.12479

24. Wiebe S, Guyatt G, Weaver B, Matijevic S, Sidwell C. Comparative responsiveness of generic and specific quality-of-life instruments. J Clin Epidemiol. (2003) 56:52-60. doi: 10.1016/s0895-4356(02)00537-1

25. Frendl DM, Ware Jr JE. Patient-reported functional health and wellbeing outcomes with drug therapy: a systematic review of randomized trials using the sf-36 health survey. Med Care. (2014) 52:439-45. doi: $10.1097 / \mathrm{mlr} .000000000000010311$

26. U.S. Food and Drug Administration. Patient-Reported Outcome Measures: Use In Medical Product Development To Support Labelling Claims. (2009). Available online at: https://www.fda.gov/Regulatory-Information/SearchFda-Guidance-Documents/Patient-Reported-Outcome-Measures-UseMedical-Product-Developmentsupport (accessed 30 Jan, 2021).

27. Tatlock S, Gober M, Williamson N, Arbuckle R. Development and preliminary psychometric evaluation of an owner-completed measure of feline quality of life. Vet J. (2017) 228:22-32. doi: 10.1016/j.tvjl.2017.10.005

28. Moher D, Liberati A, Tetzlaff J, Altman DG. PRISMA Group. Preferred reporting items for systematic reviews and meta-analyses: the PRISMA statement. PLoS Med. (2009) 6:e1000097. doi: 10.1371/journal.pmed.1000097

29. Von Elm E, Altman DG, Egger M, Pocock SJ, Gøtzsche PC, Vandenbroucke JP, et al. The strengthening the reporting of observational studies in epidemiology (STROBE) statement: guidelines for reporting observational studies. Ann Intern Med. (2007) 147:573-7. doi: 10.7326/0003-4819-147-8-200710160-00010

30. Yeowell G, Lefley-Burns D, Fatoye F, Gebrye T, Wright A, Mwacalimba K, et al. Indicators of health related quality of life in cats with degenerative joint disease: systematic review and proposal of a conceptual framework. Front Vet Sci. manuscript id: 582148 .

31. Hercock C, Pinchbeck G, Giejda A, Clegg P, Innes J. Validation of a client-based clinical metrology instrument for the evaluation of canine elbow osteoarthritis. J Small Anim Pract. (2009) 50:266-71. doi: 10.1111/j.1748-5827.2009.00765.x

32. Andersson A, Bergström A. Adaptation of the canine orthopaedic index to evaluate chronic elbow osteoarthritis in swedish dogs. Acta Vet Scand. (2019) 61:29. doi: 10.1186/s13028-019-0465-1

33. Baltzer WI, Owen R, Bridges J. Survey of handlers of 158 police dogs in New Zealand: functional assessment and canine orthopedic index. Front Vet Sci. (2019) 6:85. doi: 10.3389/fvets.2019.00085

34. Barcelos A-M, Mills DS, Zulch H. Clinical indicators of occult musculoskeletal pain in aggressive dogs. Vet Rec. (2015) 176:465. doi: 10.1136/vr.102823

35. Belshaw Z, Dean R, Asher L. You can be blind because of loving them so much: the impact on owners in the united kingdom of living with a dog with osteoarthritis. BMC Vet Res. (2020) 16:190. doi: 10.1186/s12917-020-02404-5

36. Brown DC. The canine orthopedic index. step 1: devising the items. Vet Surg. (2014) 43:232-40. doi: 10.1111/j.1532-950x.2014.12142.x

37. Brown Dc. The canine orthopedic index. step 2: psychometric testing. Vet Surg. (2014) 43:241-6. doi: 10.1111/j.1532-950x.2014.12141.x

38. Essner A, Högberg H, Zetterberg L, Hellström K, Sjöström R, Gustås $\mathrm{P}$. Investigating the probability of response bias in owner-perceived pain assessment in dogs with osteoarthritis. Top Companion Anim Med. (2020) 39:100407. doi: 10.1016/j.tcam.2020.100407

39. Essner A, Zetterberg L, Hellström K, Gustås P, Högberg H, Sjöström R Psychometric evaluation of the canine brief pain inventory in a Swedish sample of dogs with pain related to osteoarthritis. Acta Vet Scand. (2017) 59:44. doi: 10.1186/s13028-017-0319-7 
40. Gates M, Hinds H, Dale A. Preliminary description of aging cats and dogs presented to a New Zealand first-opinion veterinary clinic at end-of-life. $N Z$ Vet J. (2017) 65:313-7. doi: 10.1080/00480169.2017.1360161

41. Harris LK, Whay HR, Murrell JC. An investigation of mechanical nociceptive thresholds in dogs with hind limb joint pain compared to healthy control dogs. Vet J. (2018) 234:85-90. doi: 10.1016/j.tvjl.2017.12.012

42. Hielm-Björkman AK, Kuusela E, Liman A, Markkola A, Saarto E, Huttunen $\mathrm{P}$, et al. Evaluation of methods for assessment of pain associated with chronic osteoarthritis in dogs. J Am Vet Med Assoc. (2003) 222:1552-8. doi: 10.2460/javma.2003.222.1552

43. Knazovicky D, Helgeson ES, Case B, Gruen ME, Maixner W, Lascelles BDX. Widespread somatosensory sensitivity in naturally occurring canine model of osteoarthritis. Pain. (2016) 157:1325-32. doi: 10.1097/j.pain.0000000000000521

44. Ragetly GR, Massey L, Brown DC. Initial psychometric testing and validation of the french version of the canine brief pain inventory. Vet Anaesth Analg. (2019) 46:667-72. doi: 10.1016/j.vaa.2019.04.001

45. Reid J, Wiseman-Orr ML, Scott EM, Nolan AM. Development, validation and reliability of a web-based questionnaire to measure health-related quality of life in dogs. J Small Anim Pract. (2013) 54:227-33. doi: 10.1111/jsap.12059

46. Walton MB, Cowderoy E, Lascelles D, Innes JF. Evaluation of construct and criterion validity for the 'liverpool osteoarthritis in dogs'(load) clinical metrology instrument and comparison to two other instruments. PLoS ONE. (2013) 8:e58125. doi: 10.1371/journal.pone.0058125

47. Williams MD, Kirkpatrick AE, Griffith E, Benito J, Hash J, Lascelles BDX. Feasibility and repeatability of thermal quantitative sensory testing in normal dogs and dogs with hind limb osteoarthritis-associated pain. Vet J. (2014) 199:63-7. doi: 10.1016/j.tvjl.2013.11.003

48. Wiseman-Orr ML, Nolan AM, Reid J, Scott EM. Development of a questionnaire to measure the effects of chronic pain on health-related quality of life in dogs. Am J Vet Res. (2004) 65:1077-84. doi: 10.2460/ajvr.2004. 65.1077

49. Pettitt RA, German AJ. Investigation and management of canine osteoarthritis. In Pract. (2015) 37:1-8. doi: 10.1136/inp.h5763

50. Burke D, Lennon O, Fullen BM. Quality of life after spinal cord injury: the impact of pain. Eur J Pain. (2018) 22:1662-72. doi: 10.1002/ejp.1248

51. Claar RL, Van Tilburg MA, Abdullah B, Langer S, Sherif D, Whitehead WE, et al. Psychological distress and quality of life in pediatric crohn's disease: impact of pain and disease state. J Pediatr Gastroenterol Nutr. (2017) 65:420-4. doi: 10.1097/mpg.0000000000001549
52. Zis P, Sarrigiannis PG, Rao DG, Hewamadduma C, Hadjivassiliou M. Chronic idiopathic axonal polyneuropathy: prevalence of pain and impact on quality of life. Brain Behav. (2019) 9:E01171. doi: 10.1002/brb 3.1171

53. Epstein M, Rodan I, Griffenhagen G, Kadrlik J, Petty M, Robertson S, et al. 2015 AAHA/AAFP pain management guidelines for dogs and cats. J Am Anim Hosp Assoc. (2015) 51:67-84. doi: 10.5326/jaaha-ms-7331

54. Belshaw Z, Yeates J. Assessment of quality of life and chronic pain in dogs. Vet J. (2018) 239:59-64. doi: 10.1016/j.tvjl.2018.07.010

55. Canine arthritis management. Cam Factor. (2019). Available online at: https://caninearthritis.co.uk/wp-content/uploads/2019/12/CAM-Slides.pdf. (accessed June 08, 2021).

56. Mcmillan FD, Yeates JW. The problems with well-being terminology. In: Mcmillan FD, editor. Mental Health And Wellbeing In Animals. 2nd ed. Oxfordshire: Cab International (2019). p. 8-20. doi: $10.1079 / 9781786393401.0008$

Conflict of Interest: DB and IO are employees of company Zoetis. GC, BA, and CR's positions are funded by Zoetis, $\mathrm{LG}$ has a $\mathrm{PhD}$ scholarship which is partially funded by Zoetis. AC is an academic Principal Investigator for other projects funded or co-funded by Zoetis. The attendance of ZB and $\mathrm{HC}$ at the expert panel was compensated by Zoetis.

The remaining author declares that the research was conducted in the absence of any commercial or financial relationships that could be construed as a potential conflict of interest.

Publisher's Note: All claims expressed in this article are solely those of the authors and do not necessarily represent those of their affiliated organizations, or those of the publisher, the editors and the reviewers. Any product that may be evaluated in this article, or claim that may be made by its manufacturer, is not guaranteed or endorsed by the publisher.

Copyright (c) 2021 Roberts, Armson, Bartram, Belshaw, Capon, Cherry, Gonzalez Villeta, McIntyre, Odeyemi and Cook. This is an open-access article distributed under the terms of the Creative Commons Attribution License (CC BY). The use, distribution or reproduction in other forums is permitted, provided the original author(s) and the copyright owner(s) are credited and that the original publication in this journal is cited, in accordance with accepted academic practice. No use, distribution or reproduction is permitted which does not comply with these terms. 